\title{
Comparison of One-Dimensional and Volumetric Computed Tomography Measurements of Injected-Water Phantoms
}

\author{
Zachary H. Levine $^{1}$, H. Heather Chen-Mayer ${ }^{1}$, Adele P. Peskin ${ }^{2}$, and Adam L. Pintar ${ }^{1}$ \\ ${ }^{1}$ National Institute of Standards and Technology, \\ Gaithersburg, Maryland 20899, USA \\ ${ }^{2}$ National Institute of Standards and Technology, \\ Boulder, Colorado 80305, USA \\ zlevine@nist.gov \\ heather.chen-mayer@nist.gov \\ adele.peskin@nist.gov \\ adam.pintar@nist.gov
}

\begin{abstract}
The goal of this study was to compare volumetric analysis in computed tomography (CT) with the length measurement prescribed by the Response Evaluation Criteria in Solid Tumors (RECIST) for a system with known mass and unknown shape. We injected 2 mL to $4 \mathrm{~mL}$ of water into vials of sodium polyacrylate and into disposable diapers. Volume measurements of the sodium polyacrylate powder were able to predict both mass and proportional changes in mass within a $95 \%$ prediction interval of width $12 \%$ and $16 \%$, respectively. The corresponding figures for RECIST were $102 \%$ and $82 \%$.
\end{abstract}

Key words: CT phantom; RECIST; volumetrics.

Accepted: August 23, 2017

Published: September 20, 2017

https://doi.org/10.6028/jres.122.036

\section{Introduction}

One of the principal methods for detecting cancer is through the measurement of the growth of nodules. Arguably, the most widely accepted method is that of the Response Evaluation Criteria in Solid Tumors (RECIST) [1], which formalizes a set of rules for characterizing the size of nodules and changes in nodules based on one-dimensional (1D) measurements. Despite an early consensus statement from the International Cancer Imaging Society [2], these rules are recognized by regulatory agencies around the world including the U. S. Food and Drug Administration [3] and the European Medicines Agency [4] and have been used in more than 2000 research papers since 2010 alone. Jaffe noted that the popularity of RECIST was due to a low expectation of precision in a clinical environment and the need to demonstrate a considerable advantage to justify a more complex procedure [5].

Historically, early x-ray computed tomography (CT) measurements were displayed as a set of two dimensional (2D) images rendered onto film. Over the decades since the introduction of CT, commercial systems have shown orders of magnitude of improvement. Around the year 2000, resolution improved to the point where three dimensional (3D) measurements could be seriously considered [6].

Nevertheless, the 2009 update to the RECIST method [1] reviewed the literature of volumetric measurements and did not find sufficient evidence to go away from 1D characterizations of tumor size. 
Since that time, volumetric measurements have been shown to offer an improvement over RECIST in both phantom [7-12] and clinical [13, 14] studies. Moreover, whereas RECIST requires a minimum lesion size of $10 \mathrm{~mm}$ to be measurable, the advent of large-scale screening for small lung nodules [15], for which the lower bound in size is about $5 \mathrm{~mm}[16,17]$, underscores the necessity of developing techniques compatible with smaller nodules.

All of the phantom studies characterizing tumor size cited above were based on well-defined objects with sharp boundaries. Real tumors, however, have less well-defined boundaries, which make real tumor measurements more difficult. Our interest here is how that difficulty affects the RECIST measurement. Therefore, we studied a different model system: we created regions with known added mass but unknown shape and boundaries that were not necessarily well defined. We expected one model to be most appropriate for lung tumors, where a high-density tumor can be isolated from a low-density background. A second model represents more challenging situations.

\section{Methods and Materials}

Sodium polyacrylate is a highly absorbent polymer and is the principal ingredient of disposable diapers [18]. In this study, we obtained both sodium polyacrylate powder (Carolina Biological Supply, Burlington, North Carolina, USA) ${ }^{1}$ and disposable diapers (Proctor \& Gamble, Cincinnati, Ohio, USA). We placed from $17.0 \mathrm{~g}$ to $19.0 \mathrm{~g}$ of powder into each of 10 vials, and we found the mass using an analytical balance (Denver Instruments M-220D, Bohemia, New York, USA) with a resolution of $0.1 \mathrm{mg}$. Accuracy was ensured by comparison to a set of calibrated masses. To match the calibrated masses, it was necessary to increase the nominal reading by $1.7413 \mathrm{mg}$ and then divide by 0.98958 , i.e., a correction of about $1 \%$. The mass of each of 5 dry disposable diapers was also found.

The 15 samples were placed on a Philips Brilliant 16 CT scanner within a $1 \mathrm{~m}$ distance, so that each sample was centered, and no two samples appeared in the same slice. The scan was conducted with the settings of a tube voltage of $120 \mathrm{kV}$ and a current-time product of $200 \mathrm{mAs}$. The reconstructed images had cubic voxels $0.8 \mathrm{~mm}$ on a side. The files in Digital Imaging and Communications in Medicine (DICOM) format from the CT machine are publicly available through a server at the National Institute of Standards and Technology (NIST) [19].

After the scan, a nominal $2 \mathrm{~mL}$ aliquot of deionized water was injected into each sample using a hypodermic needle with a capacity of $5 \mathrm{~mL}$. Each sample was weighed and scanned as before. Then, for four iterations, an additional nominal $0.5 \mathrm{~mL}$ aliquot of water was injected into each sample, and the samples were rescanned. The injections were intended to be placed with the needle in the same spot each time; the precision of this operation was limited because it was done by hand. The net masses are shown in Table 1. A single scan was performed for the dry samples, but two were performed on each of the wet samples for a total of 11 scans per sample.

${ }^{1}$ Certain commercial equipment, instruments, or materials are identified in this paper to foster understanding. Such identification does not imply recommendation or endorsement by the National Institute of Standards and Technology, nor does it imply that the materials or equipment identified are necessarily the best available for the purpose. 
Table 1. The net mass (g) of added water for the vials (V2 to V11) and the diapers (D1 to D5). The header is the nominal volume of water added. All digits shown are significant. The uncertainties are negligible compared to other quantities in the study.

\begin{tabular}{rccccc}
\hline & $2 \mathrm{~mL}$ & $2.5 \mathrm{~mL}$ & $3 \mathrm{~mL}$ & $3.5 \mathrm{~mL}$ & $4 \mathrm{~mL}$ \\
\hline V2 & 2.3044 & 3.0472 & 3.5967 & 4.1724 & 4.7141 \\
V3 & 2.4064 & 3.1550 & 3.7853 & 4.3725 & 4.8758 \\
V4 & 2.2965 & 3.2248 & 3.6923 & 4.1534 & 4.6885 \\
V5 & 2.4867 & 3.2236 & 3.8599 & 4.2926 & 4.7997 \\
V6 & 2.4590 & 3.3101 & 3.7728 & 4.2180 & 4.7521 \\
V7 & 2.5123 & 3.1553 & 3.6590 & 4.1795 & 4.8307 \\
V8 & 2.4840 & 3.2818 & 3.8422 & 4.3856 & 4.9930 \\
V9 & 2.4659 & 3.1977 & 3.7157 & 4.2675 & 4.8023 \\
V10 & 2.4609 & 3.2334 & 3.7712 & 4.2784 & 4.9238 \\
V11 & 2.5534 & 3.4604 & 3.9709 & 4.5299 & 5.1449 \\
D1 & 2.0718 & 2.7942 & 3.2964 & 3.7740 & 4.2824 \\
D2 & 2.0647 & 2.7241 & 3.2714 & 3.8119 & 4.3221 \\
D3 & 2.0122 & 2.9667 & 3.4849 & 3.6611 & 4.0631 \\
D4 & 2.3633 & 3.1834 & 3.6486 & 4.2307 & 4.7174 \\
D5 & 2.1157 & 2.8537 & 3.4275 & 3.9511 & 4.4659 \\
\hline
\end{tabular}

The resulting scans were analyzed to find appropriate thresholds in Hounsfield units (HU) for the images. A value of $-200 \mathrm{HU}$ was selected for the vials and a value of $-600 \mathrm{HU}$ was selected for the diapers, values below which the dry and wet sample histograms look the same. Histograms are shown in Fig. 1 to support these choices.
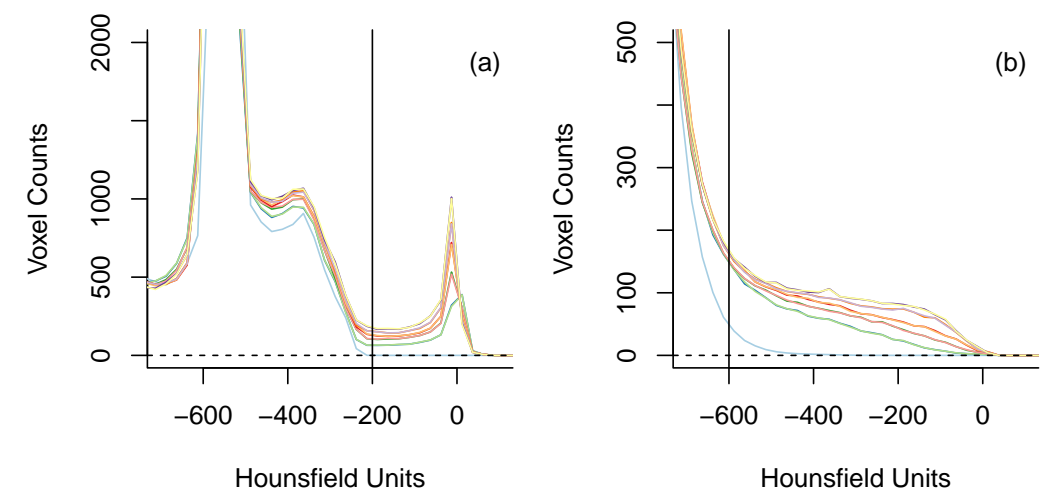

Fig. 1. Histograms of voxel counts in groups of $25 \mathrm{HU}$ for (a) powder samples, and (b) diapers. The light blue line represents the dry system. The other lines represent increasing nominal dose from $2 \mathrm{~mL}$ to $4 \mathrm{~mL}$ in steps of $0.5 \mathrm{~mL}$. The vertical lines represent the cut-offs used in this study.

Thresholds were applied to the CT images yielding one region for each sample, or a total of $15 \times 5 \times 2=150$ segmented regions, including 15 samples, 5 non-zero quantities of water, and 2 repeat scans. In practice, the repeated scans were almost identical to each other with both volumes and RECIST lengths differing from each other by less than $1 \%$ on average. Typical scans are presented in Fig. 2. 


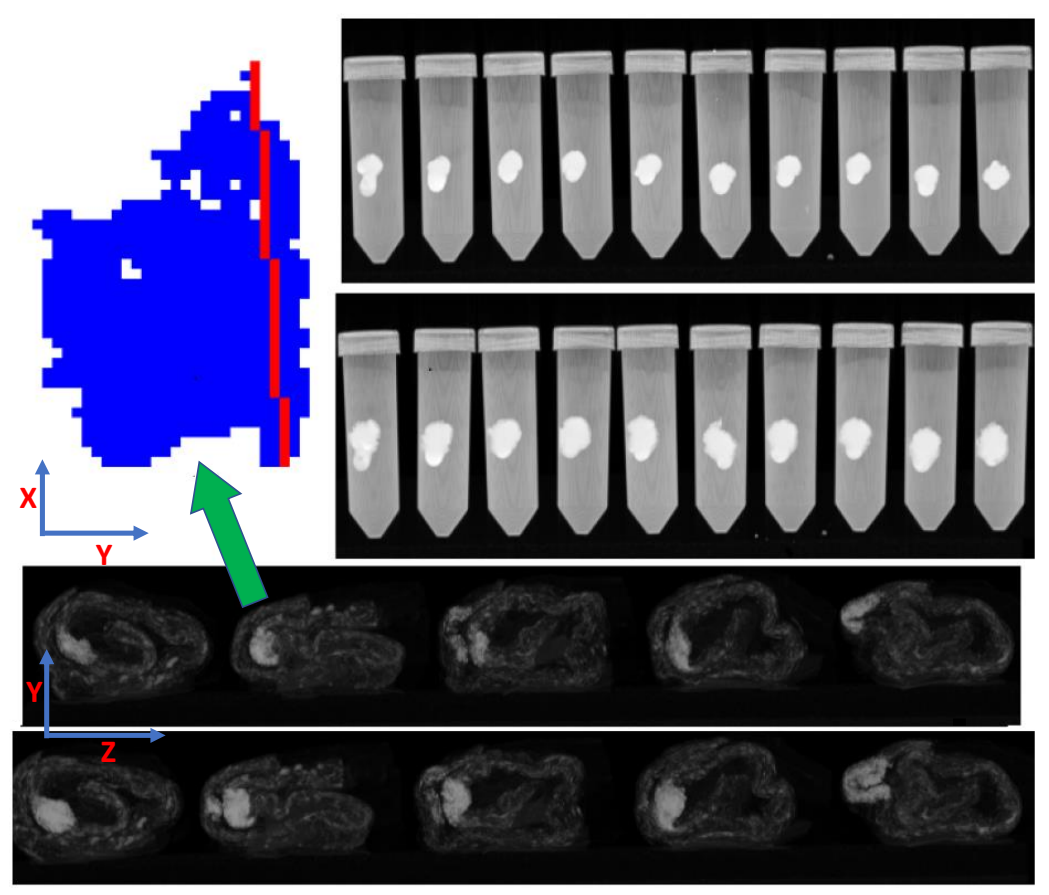

Fig. 2. Gray scale images showing orthographic sagittal projections of the vials and diapers. (The sagittal projection includes the direction of gravity $-Y$ in its natural orientation as $-y$ in the figure and the scan axis $Z$ along $x$ in the figure.) The gray scale value displayed is the maximum HU value within the column from $-1000 \mathrm{HU}$ (black) to slightly above $0 \mathrm{HU}$ (white). From left the right, the vials are V11 to V2, and the diapers are D5 to D1. Within each pair of images, the upper image represents the nominal $2 \mathrm{~mL}$ injection of water and the lower image represents the nominal $4 \mathrm{~mL}$ injection of water. Results from the first of two repeated scans are presented in each case. The segmentation in the axial plane (rotated $90^{\circ}$, so up or $Y$ in the lab is right or $x$ in the inset) is shown for one slice from diaper D4 as indicated by the green arrow. The segmented area is shown in blue or red, with the red representing the line found by the program from which the RECIST length was determined.

In addition to obtaining volumes, RECIST length measurements were made on the segmented regions in the axial slices. A typical case is shown in the inset of Fig. 2. The RECIST length is the length of the longest straight line in any single plane of acquisition that is completely included within any axial slice of the sample. In order to find the RECIST length, the program first finds the boundary of the segmented region by eroding the region and subtracting the eroded binary image from the original segmented region. Next, it considers all pairs of points on the boundary of the segmented region that are in the same slice. Then, it examines the line segment joining them to ensure that the line segment is wholly included within the segmented region. Only one region per sample-condition pair is included: we did not sum lengths of multiple "tumors" as permitted under the rules of RECIST. Although there are points in the region shown in Fig. 2 that are farther apart from each other than the RECIST length, the line segment connecting them runs through interior holes.

To reduce the risk of errors, we wrote two RECIST length and these were found to yield nearly identical results. Negligible discrepancies were attributed to the problem of classifying whether a line was entirely enclosed in a region which relied on floating-point logic.

\subsection{Predictive Model}

A predictive model taking the dependent variable to be the logarithm of mass was developed separately for the two independent variables, namely, the logarithm of volume, and the logarithm of RECIST length. In both cases, the same model form was used. Each diaper and vial was allowed its own linear trend. However, 
those trends were assumed to vary randomly around an average linear trend. Diapers and vials were assumed to have different average trends.

Some notation is introduced here to describe the model in detail. Let $m_{i s}$ be the logarithm of the mass of system $s$ after water addition $i$. For the diapers, $s=1, \ldots, 5$, and for the vials, $s=2, \ldots, 11$. Since there were 5 water additions, $i=1, \ldots, 5$. Let $v_{i s r}$ be the logarithm of the volume, shifted by a central value, from scan $r=1,2$ of system $s$ after water addition $i$, and let $\ell_{i s r}$ be the equivalent for the RECIST length. The shift is important so that the intercepts are not extrapolations. Let $v_{i s}$ be the true logarithm of the volume for system $s$ after water addition $i$, and let $\lambda_{i s}$ be the equivalent for the RECIST length. It is necessary to distinguish between the true unobserved volume and RECIST length and their measured values because of scan-to-scan variability. The model is then

$$
\begin{aligned}
m_{i s} & \sim \operatorname{Normal}\left(b_{0 s}+b_{1 s} v_{i s}, \sigma^{2}\right), \\
b_{0 s} & \sim \operatorname{Normal}\left(\beta_{0}, \sigma_{0}^{2}\right), \\
b_{1 s} & \sim \operatorname{Normal}\left(\beta_{1}, \sigma_{1}^{2}\right), \\
v_{i s r} & \sim \operatorname{Normal}\left(v_{i s}, \sigma_{v}^{2}\right) .
\end{aligned}
$$

The model based on the RECIST length simply replaces $v$ and $v$ by $\ell$ and $\lambda$, respectively. The model for mass ratios vs. volume or RECIST length ratios uses the same form except the intercept parameters $b_{0 s}, \beta_{0}$, and $\sigma_{0}$ are omitted. If a given observation were considered in the opposite order, the log of the ratio would simply change sign for both independent and dependent variables. The model is therefore constrained to be an odd function of the logarithm of the independent variable; as a corollary, the point $(1,1)$ must appear in the fitting lines for the change ratios.

The model was fitted using the Bayesian inference paradigm [20]; thus, the joint posterior distribution of $b_{0 s}, b_{1 s}, v_{i s}, \sigma, \beta_{0}, \sigma_{0}, \beta_{1}, \sigma_{1}$, and $\sigma_{v}$ was calculated. The necessary ingredients were a likelihood and a prior distribution. Equation (1) defines the likelihood and specifies prior distributions for $b_{0 s}$ and $b_{1 s}$. Most of the remaining parameters were assigned improper flat prior distributions with the intention that their impact on the final result would be negligible. The exceptions were $\sigma_{0}$ and $\sigma_{1}$. They were assigned half-normal distributions with location parameter equal to zero and scale parameters given in Table 2 . Computations were carried out using $\mathrm{R}$ [21] and the R package rstan [22, 23].

Table 2. Parameters used for prior distributions. For the model of the changes in these quantities, $\sigma_{0}$ is omitted.

\begin{tabular}{lccc}
\hline & & $\sigma_{0}$ & $\sigma_{1}$ \\
\hline Powder & $V$ & 0.45 & 1 \\
Diaper & $V$ & 0.60 & 1 \\
Powder & $\ell$ & 0.80 & 3 \\
Diaper & $\ell$ & 1.10 & 3 \\
\hline
\end{tabular}

\section{Results}

Our results for water injected into sodium polyacrylate powder are shown in Fig. 3. Whether we consider the prediction of mass or the change in mass, knowledge of the volume or change in volume, respectively, is a far better predictor than the corresponding RECIST lengths. The gray areas in Fig. 3 are $95 \%$ prediction regions for expected measurements from a new vial of powder that was not part of the original experiment. Narrower intervals indicate better predictive ability. Since the widths of the intervals vary across volume, volume change, RECIST, and RECIST change, their widths are summarized as the average value of $2\left(B_{u}-B_{\ell}\right) /\left(B_{u}+B_{\ell}\right)$ where $B_{u}$ and $B_{\ell}$ are the upper and lower bounds, respectively. For Fig. 3, these values are (a) $12 \%$, (b) $102 \%$, (c) $16 \%$, and (d) $82 \%$. The volume measurements (a) and (c) outperform the RECIST length measurements (b) and (d) by a factor of five or more in predicting both mass and change in mass. 


\section{Journal of Research of National Institute of Standards and Technology}
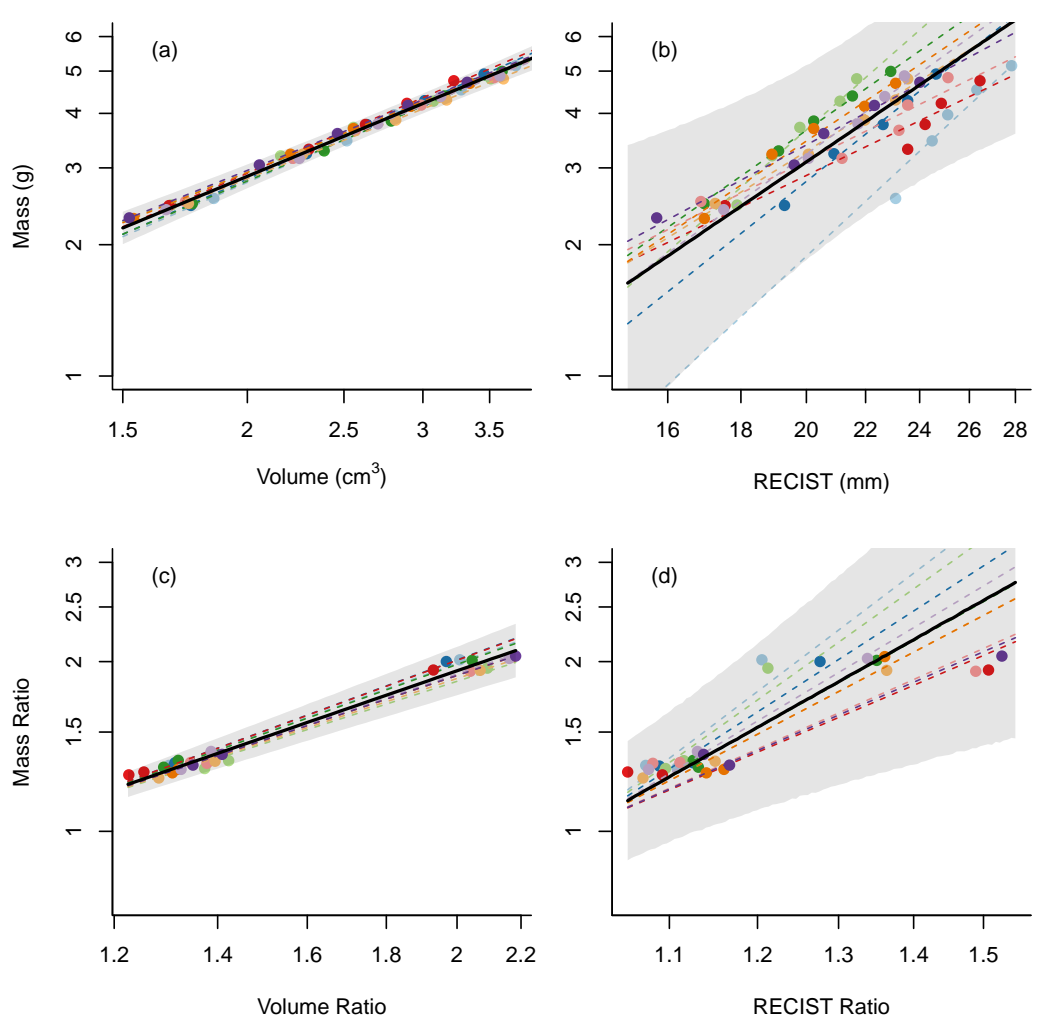

Fig. 3. (a) Mass vs. volume of largest thresholded region for water injected into 10 vials; (b) mass vs. length as measured by RECIST for the same thresholded regions with the same $y$ axis as in part (a); (c) change in mass vs. change in volume for the vials with $m_{5 s}-m_{1 s}, m_{4 s}-m_{2 s}$, and $m_{5 s}-m_{3 s}$, on the $y$ axis, and $\left\langle v_{5 s}-v_{1 s}\right\rangle,\left\langle v_{4 s}-v_{2 s}\right\rangle$, and $\left\langle v_{5 s}-v_{3 s}\right\rangle$, on the $x$ axis, where the angle brackets indicate averaging over the two replicate scans; (d) change in mass vs. change in RECIST length for the vials with the same $y$ axis as in part (c). In all cases, the fit lines are given as described in the text, and the gray regions represent $95 \%$ prediction intervals for the fit lines. Dots of a given color belong to the same sample.

Our results for water injected into disposable diapers are shown in Fig. 4. We did not attempt to give prediction bounds for a new diaper which was not part of the original experiment as we had done for the vials. Nevertheless, for each diaper the expected positive linear trend between mass and volume is clearly present, and captured by the model. In contrast, blending the behavior with the RECIST length as the predictor leads to only a weak trend. For example, the average errors for the mass predictions are (a) $2.7 \%$ and (b) $20 \%$ with the volume and the RECIST length as the independent variables, respectively, and (c) $3.4 \%$ and (d) $47 \%$ for the predictions of changes in mass with changes in volume or changes in the RECIST length as the independent variable, respectively, showing that the volumetric measurement again outperforms the RECIST length by at least a factor of five in this heterogeneous environment. In the case of change in mass, the trivial model of no mass change regardless of the volume or the RECIST length leads to a $66 \%$ average error which is not a great deal worse than the $47 \%$ in the RECIST model.

The magnitude of the slopes in Fig. 3 and Fig. 4 have an interpretation. Assuming equal density, we expect the mass to grow linearly with the volume, so the slope on the log plot should be 1 . For the case of the RECIST length $\ell$, if the regions are growing isotropically, then the mass should be proportional to $\ell^{3}$. As summarized in Table 3, this expectation is well met for volume, with slopes of 0.95 to 1.08 for powder and diaper samples, respectively. In contrast, the agreement with the RECIST model is poor to fair. This suggests that the changes in the shapes of the injected-water regions are not well described by an isotropic expansion model. 


\section{Journal of Research of National Institute of Standards and Technology}
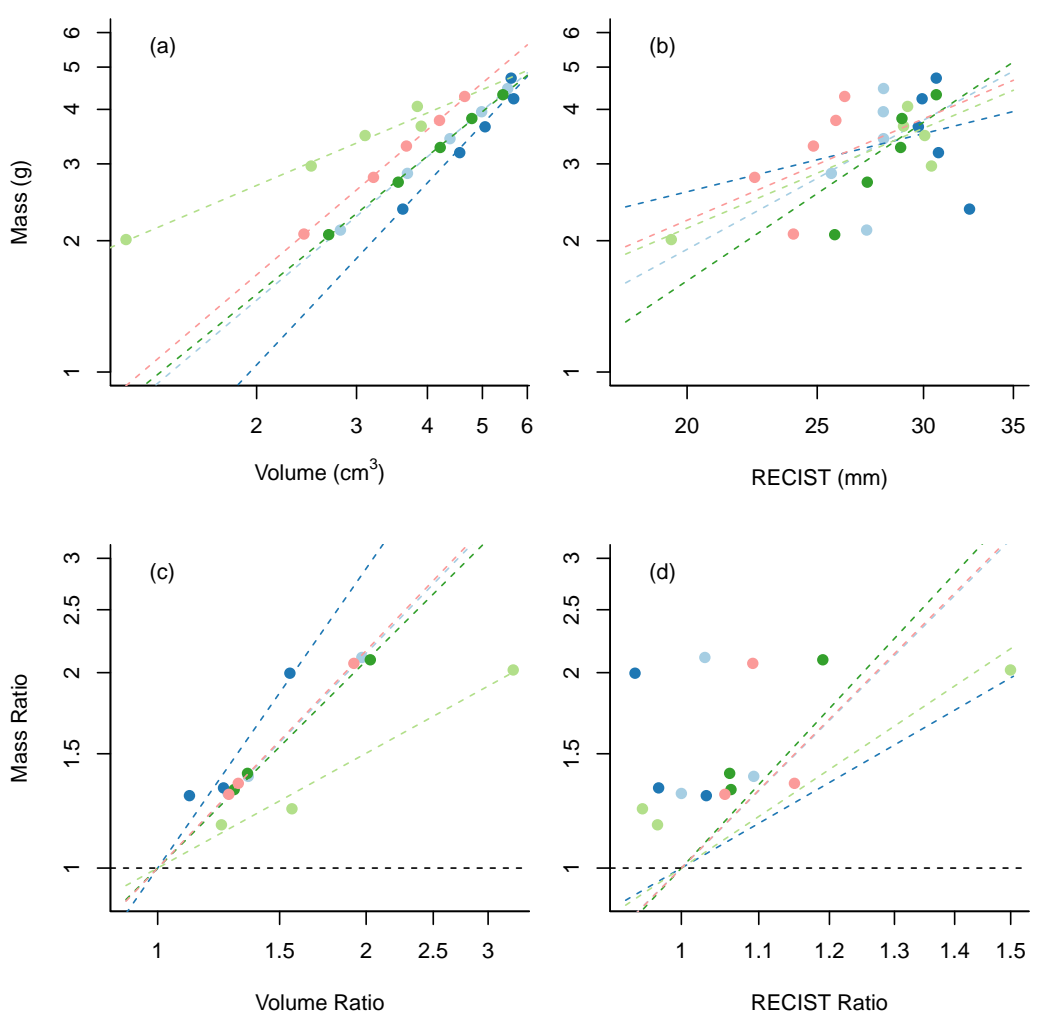

Fig. 4. (a) Mass vs. volume of largest thresholded region for water injected into 5 diapers; (b) mass vs. length as measured by RECIST for the same thresholded regions; (c) change in mass vs. change in volume for the vials, with the formula given in Fig. 3 caption; (d) change in mass vs. change in RECIST length for the diapers. The fit lines are described in the text. Dots of a given color belong to the same sample.

Table 3. Average slopes from figures for the logarithm of volume $V$ or the RECIST length $\ell$ or ratios of these quantities. The column labeled "ideal" refers to the value within the isotropic expansion model.

\begin{tabular}{lcccc}
\hline & & Slope & Ideal & Fig. \\
\hline Powder & $\ln V$ & 0.95 & 1 & $3 \mathrm{a}$ \\
Powder & $\ln V_{2} / V_{1}$ & 0.95 & 1 & $3 \mathrm{c}$ \\
Diaper & $\ln V$ & 1.04 & 1 & $4 \mathrm{a}$ \\
Diaper & $\ln V_{2} / V_{1}$ & 1.08 & 1 & $4 \mathrm{c}$ \\
Powder & $\ln \ell$ & 2.23 & 3 & $3 \mathrm{~b}$ \\
Powder & $\ln \ell_{2} / \ell_{1}$ & 2.32 & 3 & $3 \mathrm{~d}$ \\
Diaper & $\ln \ell$ & 1.43 & 3 & $4 \mathrm{~b}$ \\
Diaper & $\ln \ell_{2} / \ell_{1}$ & 2.47 & 3 & $4 \mathrm{~d}$ \\
\hline
\end{tabular}

\section{Discussion}

In studying the absorption of water in sodium polyacrylate and in disposable diapers, we have two model systems, the former is more homogeneous, and the latter is more heterogeneous. In both cases, we create objects for which the exact boundaries are arbitrary to some extent. 
It is perhaps surprising that in certain cases in the diapers, the RECIST length decreases when water is added. Recall that the RECIST length is the longest diameter that fits in a region that is in a single axial slice. If the process of adding water strictly expanded the regions, one would expect a region with less water to be a subset of the corresponding region with more water, and the region with more water would have the larger RECIST length. However, in practice, the addition of water rearranges the existing water, and the strict expansion assumption does not hold. Hence, there is no problem in principle with a negative change in the RECIST length measurement being associated with a positive change in water mass.

The model in Eq. (1) is useful because it automatically pools information when appropriate. For instance, if the diapers or vials are basically identical to one another their estimated individual trends will collapse to the estimated average. In this case, $\sigma_{0}$ and $\sigma_{1}$ both approach zero. An example is the vials using volume as the independent variable as in Fig. 3a. On the other hand, if individual diapers or vials are disparate from one another their estimated individual trends will deviate substantially from the estimated average and $\sigma_{0}$ or $\sigma_{1}$ will grow large. An example is the diapers using volume as the independent variable, such as in Fig. 4a.

The impact of the prior distribution parameters $\sigma_{0}$ and $\sigma_{1}$ given in Table 2 on the final results varied. For instance, their impact was negligible for the powder-volume combination, but notable for the diaper-RECIST length combination. In general, when the model does not describe the data very well, the fitting parameters depend more strongly on the assumptions of the model.

\section{Conclusions}

One of the differences between typical x-ray phantoms and real tumors is the difficulty of describing the boundary of the tumors. Tumors are sometimes well-defined ("solid tumors") and sometimes less so ("ground glass tumors"). Here, we studied two related systems in which the boundaries were challenging to define. We injected water into sodium polyacrylate powder and into disposable diapers. Although we did not know exactly where the water went in these systems, we did know the total mass of added water to high accuracy. The water-in-powder system is less heterogeneous than the water-in-diaper system, because the diaper has compartments and stitching holding the powder into a useful consumer product. Perhaps it is not surprising that the predictions are better for both volumetric and RECIST length in the water-in-powder system. However, our main focus was on volumetric measurement methods vs. the RECIST length. We found that volumetric method outperforms the length of the longest diameter as determined by the rules of RECIST by at least a factor of five in its ability to predict the ground truth of mass.

\section{Acknowledgments}

The authors would like to thank Brian Zimmerman for operation of the CT, as well as Eliot Siegel, Joseph Chen, and Jeffrey Gunn for helping us conduct a preliminary version of this study.

\section{References}

[1] Eisenhauer EA, Therasse P, Bogaerts J, Schwartz LH, Sargent D, Ford R, Dancey J, Arbuck S, Gwyther S, Mooney M, Rubenstein L, Shankar L, Dodd L, Kaplan R, Lacombe D, Verweij J (2009) New response evaluation criteria in solid tumors: Revised RECIST guideline (version 1.1). European Journal of Cancer 45(2):228-247. https://doi.org/10.1016/j.ejca.2008.10.026.

[2] Husband JE, Schwartz LH, Spencer J, Olliver L, King DM, Johnson R, Reznek R (2004) Evaluation of the response to treatment of solid tumors - A consensus statement of the International Cancer Imaging Society. British Journal of Cancer 90(12):2256-2260. https://doi.org/10.1038/sj.bjc.6601843.

[3] US Department of Health and Human Services, Food and Drug Administration, Center for Drug Evaluation and Research, Center for Biologics Evaluation and Research (2015) Clinical Trial Imaging Endpoint Process Standards: Guidance for Industry, Draft Guidance. http://www.fda.gov/downloads/drugs/guidancecomplianceregulatoryinformation/guidances/ucm268555.pdf. Retrieved 19 January 2017. 
[4] European Medicines Agency (2013) Guideline on the Evaluation of Anticancer Medicinal Products in Man, EMA/CHMP/205/95/Rev.4. http://www.ema.europa.eu/docs/en_GB/document_library/Scientific_guideline/2013/01/WC500137128.pdf. Retrieved 19 January 2017.

[5] Jaffe CC (2006) Measures of response: RECIST, WHO, and new alternatives. Journal of Clinical Oncology 24(20):3245-3251. https://doi.org/10.1200/JCO.2006.06.5599.

[6] Yankelevitz DF, Reeves AP, Kostis WJ, Zhao B, Henschke CI (2000) Small pulmonary nodules: Volumetrically determined growth rates based on CT evaluation. Radiology 217(1):251-256. https://doi.org/10.1148/radiology.217.1.r00oc33251.

[7] Levine ZH, Borchardt BR, Brandenburg NJ, Clark CW, Muralkrishnan B, Shakarji CM, Chen JJ, Siegel EL (2010) RECIST versus volume measurement in medical CT using ellipsoids of known size. Optics Express 18(8):8151-8159. https://doi.org/10.1364/OE.18.008151.

[8] Levine ZH, Galloway BR, Peskin AP, Heussel CP, Chen JJ (2011) Tumor volume measurement errors of RECIST studied with ellipsoids. Medical Physics 38(5):2552-2557. https://doi.org/10.1118/1.3577602.

[9] Gavrilides MA, Li Q, Zeng R, Meyers KJ, Sahiner B, Petrick N (2013) Minimum detectable change in lung nodule volume in a phantom CT study. Academic Radiology 20(11):1364-1370. https://doi.org/10.1016/j.acra.2013.08.019.

[10] Petrick N, Kim HJG, Clunie D, Borradalle K, Ford R, Zeng R, Gavrilides MA, McNitt-Gray MF, Lu ZQJ, Fenimore C, Zhao B, Buckler AJ (2014) Comparison of 1D, 2D, and 3D nodule sizing methods by radiologists for spherical and complex nodules on thoracic CT phantom images. Academic Radiology 21(1):30-40. https://doi.org/10.1016/j.acra.2013.09.020.

[11] Li Q, Gavrilides MA, Zeng RP, Meyers KJ, Sahiner B, Petrick N (2015) Volume estimation of low-contrast lesions with CT: A comparison of performances from a phantom study, simulations and theoretical analysis. Physics in Medicine and Biology 60(2):671-688. https://doi.org/10.1088/0031-9155/60/2/671.

[12] Athelogou M, Kim HJ, Dima A, Obuchowski N, Peskin A, Gavrilides MA, Petrick N, Saiprasad G, Colditz DC, Beaumont H, Oubel E, Tan YQ, Zhao BS, Kuhnigk JM, Motlz JH, Orieux G, Gillies RJ, Gu YH, Mantri N, Goldmacher G, Zhang LD, Vega E, Bloom M, Jarecha R, Soza G, Tietjen C, Takeguchi T, Yamagata Y, Peterson S, Masoud O, Buckler AJ (2016) Algorithm variability in the estimation of lung nodule volume from phantom CT scans: Results of the QIBA 3A public challenge. Academic Radiology 23(8):640-952. https://doi.org/10.1016/j.acra.2016.02.018.

[13] Mozley PD, Schwartz LH, Bentsen C, Zhao B, Petrick N, Buckler AJ (2010) Change in lung tumor volume as a biomarker of treatment response: A critical review of the evidence. Annals of Oncology 21(9):1751-1755. https://doi.org/10.1093/annonc/mdq051.

[14] Rothe JH, Grieser C, Lehmkuhl L, Schnapauff D, Fernandez CP, Maurer MH, Mussier A, Hamm B, Denecke T, Steffen IG (2013) Size determination and response assessment of liver metastases with computed tomography: Comparison of RECIST and volumetric algorithms. European Journal of Radiology 82(11):1831-1839. https://doi.org/10.1016/j.ejrad.2013.05.018.

[15] US Preventive Services Task Force (2013) Lung Cancer: Screening. http://www.uspreventiveservicestaskforce.org/Page/Document/UpdateSummaryFinal/lung-cancer-screening. Retrieved 28 July 2016.

[16] Henschke CI, Yankelevitz DF, Libby DM, Pasmantier MW, Smith JP, Miettinen OS (2006) Survival of patients with stage I lung cancer detected on CT screening. New England Journal of Medicine 355(17):1763-1761. https://doi.org/10.1056/NEJMoa060476.

[17] Aberle DR, Adams AM, Berg CD, Black WC, Clapp JD, Fagerstrom RM, Gareen IF, Gatsonis C, Marcus PM, Sicks JD (2011) Reduced lung-cancer mortality with low-dose computed tomographic screening. New England Journal of Medicine 365(5):395-409. https://doi.org/10.1056/NEJMoa1102873.

[18] Parks LR (1981) Cross-linked sodium polyacrylate absorbent, U.S. Patent 4295987 A.

[19] DICOM files are available on the NIST server. https://doi.org/10.18434/M31592. Retrieved 25 August 2017.

[20] Gelman A, Carlin JB, Stern HS, Dunson DB, Vehtari A, Rubin DB (2014) Bayesian Data Analysis (CRC Press, Boca Raton, FL), 3rd Ed.

[21] R Core Team (2016) R: A Language and Environment for Statistical Computing (R Foundation for Statistical Computing, Vienna, Austria). https://www.R-project.org/. Retrieved 18 September 2017.

[22] Hoffman MD, Gelman A (2014) The no-U-turn sampler: Adaptively setting path lengths in Hamiltonian Monte Carlo. Journal of Machine Learning Research 15:1593-1623.

[23] Carpenter B, Gelman A, Hoffman MD, Lee D, Goodrich B, Betancourt M, Brubaker MA, Guo JQ, Li P, Riddell A (2017) Stan: A probabilistic programming language. Journal of Statistical Software 76(1):1-29. https://doi.org/10.18637/jss.v076.i01.

\footnotetext{
About the authors: Zachary Levine is a Physicist specializing in light-matter interactions. H. Heather Chen-Meyer is a Chemist with expertise in ionizing radiation. Adele Peskin is a Computer Scientist concentrating on image processing. Adam Pintar is a Mathematical Statistician whose work often centers on likelihood and Bayesian methods. The National Institute of Standards and Technology is an agency of the U.S. Department of Commerce.
} 